\title{
The Fabrication of Hierarchical Carbon Nanotube/carbon fibre/polyethylene Composites via Twin Screw Extruder
}

\author{
Chao $\mathrm{Hu}^{1}$, Xinwen Liao ${ }^{1}$, Qinghua Qin ${ }^{1, a}$ and Gang Wang ${ }^{2}$ \\ ${ }^{1}$ Research Schoole of Engineering, The Australian National University, Canberra, Australia \\ ${ }^{2}$ Institute of Materials Science, Shanghai University, Shanghai, China
}

\begin{abstract}
In this research, the hierarchical carbon nanotube/carbon fibre/polyethylene (CNT/CF/PE) composites were fabricated via the conventional twin-screw extrusion technique. For this multi-component composite, $1 \%$ wt CNTs were uniformly coated onto the surface of CF by using spray coating method. The effect of CNTs and CFs as reinforcing fillers on the mechanical properties was investigated through mechanical test and Scanning Electron Microscopy (SEM) characterization. It has been found that with the content increase of CFs (i.e. $0 \% \mathrm{wt}, 5 \% \mathrm{wt}, 10 \% \mathrm{wt}, 15 \% \mathrm{wt}, 20 \% \mathrm{wt}, 25 \% \mathrm{wt}$ ), both of tensile strength and tensile modulus exhibited an upward trend. Moreover, $\mathrm{CF} / \mathrm{PE}$ composites with CNT coatings have higher mechanical performance than the counterparts without CNT coatings. The SEM results demonstrated that with the spray coating of CNTs onto CFs, the surface roughness of CFs was increased, thus contributing to the improvement of interfacial bonding between the reinforcement $(\mathrm{CFs})$ and the matrix $(\mathrm{PE})$.
\end{abstract}

\section{Introduction}

The main advantages of using high performance fibres as reinforcement in composite manufacturing encompass higher tensile modulus, higher strength and stiffness, and lower density. However, apart from intrinsic properties of the reinforcement and the matrix, the entire performance of the resultant composite is also, to a large extent, modulated by the interfacial properties between these two phases. Excellent interfacial bonding contributes to the efficient load transfer from the matrix to the reinforcement, which largely avoids the occurrence of stress concentrations and enhances the overall mechanical performance[1]. Moreover, the interfacial strength of the fibre-matrix entities plays a significant role in fracture toughness, compressive strength and strain to failure, fatigue resistance, impact strength and damage initiation threshold[2].

With respect to the in-plane properties of laminated composite materials, the fibre reinforcement can provide sufficient strength for many practical applications, while for the out-of-plane (i.e. through thickness) properties, the matrix usually becomes the main restraining factor for the holistic enhancement of fibre-reinforced laminated composites[3]. Existing techniques such as z-pinning, stitching and braiding have been applied for the interfacial improvement[4]. However, these methods not only increase the additional manufacturing complexity and cost, but simultaneously compromise the in-plane strength. Therefore, fibre or matrix modifications via introducing nanoscale additives

\footnotetext{
${ }^{a}$ Corresponding author : qinghua.qin@anu.edu.au
} 
open up a new avenue for the improvement of interfacial properties without increasing the overall volume of the composite. Numerous researches have been carried out in recent years in developing nano-engineered hierarchical composite materials where nanoscale additives (e.g. alumina, titania and silica) are finely integrated with conventional fibres[5-7].

Of all these nanofillers, the exploitation of carbon nanotubes (CNTs) - seamless cylinders of one or more layers of graphene with open or closed ends - is undoubtedly a big current interest in creating hierarchically reinforced composite structures[8]. Based on their unique spatial structures and remarkable strength and modulus, substantial improvements for the fibre-matrix interfacial strength and matrix dominated through-thickness performance have been achieved in glass fibre and carbon fibre reinforced composites, such as impact strength, fracture toughness, interfacial shear strength and fatigue life[9, 10]. In general, CNTs engineered hierarchical composites can be fabricated via two approaches: incorporation of CNTs into the matrix or direct adherence of CNTs onto the reinforcing fibre surface[11]. The first approach is normally limited to low concentrations of the nanofillers. With the loading increase of CNTs, the matrix will become increasingly viscose, leading to the difficulty of efficient dispersion of CNTs into the resin and sufficient filtration of the matrix into fabric reinforcement[12]. Another drawback existing in the addition of CNTs into the matrix is the limited capacity of interfacial bonding between the fibre surface and the nanoscale reinforcement. Thus, more researchers have shifted the related study to directly incorporate CNTs into fibre tows or fabrics. Four different strategies can be identified for the direct integration with reinforcement phase: (1) epitaxial growth of CNTs from the fibre surface via chemical vapor deposition (CVD); (2) electrophoretic deposition of CNTs on the fibre surface; (3) chemical bonding between group-modified CNTs and fibres; (4) spray coating of fibres and fabrics by CNTs contained solutions[13-16].

However, some of these techniques have internal disadvantages for the CNTs deposition onto fibres and fabrics. Due to the high processing temperature and the reaction of the catalyst with fibre surface in the CVD process, both of the existing sizings around fibres during their manufacture and the tensile strength of the resultant composite are damaged, let alone the less flexibility in tuning the adhesion between CNTs and fibres[17]. The operational principle underlying the electrophoresis deposition technique also necessitates the possession of electric conductivity for fabric substrates, which to a certain extent limits the application of this method[18]. Furthermore, the complicated steps and cost involved in some of these techniques largely decrease the productivity and their potential for scale-up. Compared to these methods, the CNTs deposition by coating approach shows a great promise because of its easy operation and no harm to fibres (even able to heal surface defects and improve tensile properties) $[19,20]$. It also does not need complex chemical modifications for pristine CNTs during the coating process, which are usually the prerequisite for other deposition techniques. By spray coating method, CNTs can be localized onto damage prone areas rather than deposited throughout the entire composite structure, enabling the fabrication of nano-engineered hierarchical composite systems with tunable CNTs distribution for improved material performance[21]. Consequently, the spray coating technology for CNTs deposition onto fibrous reinforcement has the most feasibility for large-scale industrial production.

In this research, carbon nanotubes with remarkable mechanical, electrical and thermal properties will be employed to create a hierarchical CNT-based high performance carbon fibre-reinforced composite. Since no report has been published concerning the effects of CNT coating on the extrusion of carbon fibre reinforced composites, the spray coating method for CNTs deposition onto carbon fibres will be investigated as well as the influence of the twin-screw extrusion on the resultant interfacial properties. The final nano-engineered composites will also be estimated via mechanical and morphological characterizations.

\section{Experimental}

\subsection{Materials}


The HDPE pellets used in this research were purchased from Qenos (Melbourne, Australia) with the tradename of Alkatane GE 7660. The mass density and average pellet size were $0.959 \mathrm{~g} / \mathrm{cm}^{3}$ and 4.6 $\mathrm{mm}$, respectively. The reinforcer introduced into the composite was chopped polyacrylonitrile-derived CF from ACP Composites (Livermore, USA). Its average length was $3 \mathrm{~mm}$. The commercial functionalized MWCNTs with hydroxyl groups with average diameter of $20 \mathrm{~nm}$ were manufactured by chemical vapor deposition technique.

\subsection{Spray coating}

Before spray coating, $1 \mathrm{mg} / \mathrm{ml} \mathrm{CNT/ethanol} \mathrm{suspension} \mathrm{was} \mathrm{prepared} \mathrm{by} \mathrm{using} \mathrm{an} \mathrm{ultrasonic} \mathrm{water}$ bath (WUA-A09H, Thermoline Scientific, Austalia) to eliminate the agglomeration of CNTs. The ultrasonication was experienced for $10 \mathrm{~min}$ with the frequency of $35 \mathrm{~Hz}$. Due to the light weight of chopped CFs, they were soaked with ethanol in advance to prevent them blowing during the spray coating process. After that, the ultrasonic treated CNT/ethanol suspension was loaded and spray deposited onto the surface of CFs with the $1 \%$ concentration by utilizing an air spray gun (Voilamart, Australia). The pressure was set at $20 \mathrm{psi}$ with the flow rate of $0.2 \mathrm{ml} / \mathrm{s}$ from a $10 \mathrm{~cm}$ working distance. The CNT-coated CFs were dried for $24 \mathrm{~h}$ prior to the commencement of composite fabrication.

\subsection{Extrusion and injection molding}

The fillers (i.e. uncoated CFs or CNT-coated CFs) and matrix (i.e. HDPE) with varying ratios were fed into a laboratory scale miniature twin-screw extruder (WLG10, China), where the screw speed and barrel temperature were set at $60 \mathrm{rpm}$ and $200^{\circ} \mathrm{C}$, respectively. The mixtures were compounded in the extruder for 5 mins and then transferred to the injection molding machine (WZS10D, China). During the injection molding process, the pressure was maintained at 980 bars for $10 \mathrm{~s}$. The cylinder temperature was set at $200{ }^{\circ} \mathrm{C}$ and the mold temperature was $60{ }^{\circ} \mathrm{C}$. Samples with standard dumbbell shape were fabricated for the tensile test. For all these samples, they can be categorized into two groups:

1. PE, $5 \% \mathrm{CF} / \mathrm{PE}, 10 \% \mathrm{CF} / \mathrm{PE}, 15 \% \mathrm{CF} / \mathrm{PE}, 20 \% \mathrm{CF} / \mathrm{PE}$ and $25 \% \mathrm{CF} / \mathrm{PE}$

2. $\mathrm{PE}, \quad 5 \% \mathrm{CNT} / \mathrm{CF} / \mathrm{PE}, \quad 10 \% \mathrm{CNT} / \mathrm{CF} / \mathrm{PE}, \quad 15 \% \mathrm{CNT} / \mathrm{CF} / \mathrm{PE}, \quad 20 \% \mathrm{CNT} / \mathrm{CF} / \mathrm{PE}$ and $25 \%$ $\mathrm{CNT} / \mathrm{CF} / \mathrm{PE}$

\subsection{Tensile properties}

According to the GB/T 1447 testing standard, tensile tests were performed on a universal testing machine (MTS CMT 5205, China) equipped with a load cell of $100 \mathrm{kN}$ at a constant crosshead speed of $5 \mathrm{~mm} / \mathrm{min}$. At least 3 parallel samples for each composition were recorded for the following tensile strength and modulus analysis.

\subsection{Scanning electron microscopy (SEM)}

The surface morphology of uncoated CFs, CNT-coated CFs and the facture morphology of composites were observed by employing a scanning electron microscope (SEM, SU-1510, HITACHI, Japan) with the acceleration voltage of $15 \mathrm{kV}$. All samples were sputter-coated with gold before analysis so as to increase the electric conductivity and SEM resolution.

\section{Results and discussions}

\subsection{Tensile properties of the composites}


Table 1 listed the mechanical properties of the PE reinforced with various weight percentages of uncoated CFs and CNTs-coated CFs. In order to investigate the mechanical performance of the CNTcoated $\mathrm{CF} / \mathrm{PE}$ composite and untreated $\mathrm{CF} / \mathrm{PE}$, pure $\mathrm{PE}$ was also tested as a control. The composite tensile strength increased along with growing amount of CFs and finally reached at the maximum value for 25 wt. \% CFs content. Compared to the pure PE, the tensile strength had improved by 94.95\% and $75.37 \%$ when it was reinforced with CNTs-coated and uncoated CNTs respectively. Meanwhile, for the same percentage content of CFs, CF/PE composites with CNT coatings had higher tensile strength than the counterparts without CNT coatings. Likewise, with the increase of CFs content, tensile modulus of the uncoated $\mathrm{CF} / \mathrm{PE}$ composite increased constantly and reached the maximum value at $25 \mathrm{wt}$ \% CNTs-coated CF content. Compared to the pure PE, the tensile modulus of PE composite with 25 wt. \% uncoated CFs and CNTs-coated CFs had been improved by $110.11 \%$ and $1.15 \%$ respectively. Meanwhile, for the same content of CFs, the tensile modulus of CNTs-coated composites had higher tensile modulus than uncoated counterparts. Conversely, the elongation at break point corresponding to the maximum displacement decreased with the increase of CF content, which indicated that the composites became increasingly brittle with the content increase of CFs. Besides, the comparison of elongation at break point between $\mathrm{CF} / \mathrm{PE}$ and $\mathrm{CNT} / \mathrm{CF} / \mathrm{PE}$ composites demonstrated that $\mathrm{CF} / \mathrm{PE}$ with $\mathrm{CNT}$-coating was more brittle than that of without CNT-coating.

Table 1. Tensile properties of pure PE and composites

\begin{tabular}{|c|c|c|c|}
\hline \multirow{2}{*}{ Specimen } & \multicolumn{3}{|c|}{ Tensile Properties } \\
\cline { 2 - 4 } & $\begin{array}{c}\text { Tensile } \\
\text { Strength } \\
(\mathbf{M P a})\end{array}$ & $\begin{array}{c}\text { Tensile } \\
\text { Modulus } \\
(\mathbf{M P a})\end{array}$ & $\begin{array}{c}\text { Elongation } \\
\text { at break (\%) }\end{array}$ \\
\hline $\mathrm{PE}$ & 30.445 & 278.621 & $52.231 \%$ \\
\hline $5 \% \mathrm{CF} / \mathrm{PE}$ & 31.023 & 398.963 & $51.175 \%$ \\
\hline $5 \% \mathrm{CNT} / \mathrm{CF} / \mathrm{PE}$ & 33.264 & 417.297 & $45.024 \%$ \\
\hline $10 \% \mathrm{CF} / \mathrm{PE}$ & 36.215 & 503.415 & $32.421 \%$ \\
\hline $10 \% \mathrm{CNT} / \mathrm{CF} / \mathrm{PE}$ & 39.551 & 536.016 & $28.278 \%$ \\
\hline $15 \% \mathrm{CF} / \mathrm{PE}$ & 42.177 & 540.048 & $19.270 \%$ \\
\hline $15 \% \mathrm{CNT} / \mathrm{CF} / \mathrm{PE}$ & 45.122 & 578.612 & $15.286 \%$ \\
\hline $20 \% \mathrm{CF} / \mathrm{PE}$ & 47.495 & 550.552 & $14.667 \%$ \\
\hline $20 \% \mathrm{CNT} / \mathrm{CF} / \mathrm{PE}$ & 52.753 & 2723.125 & $5.871 \%$ \\
\hline $25 \% \mathrm{CF} / \mathrm{PE}$ & 53.391 & 585.408 & $12.890 \%$ \\
\hline $1.25 \% \mathrm{CNT} / \mathrm{CF} / \mathrm{PE}$ & 59.354 & 3486.111 & $4.688 \%$ \\
\hline
\end{tabular}

The CNT-coated CF reinforced PE composite exhibited significant enhancement in tensile properties, which was higher than the uncoated counterparts. This is because the interface bonding between CFs and PEs was enhanced by the spray coating surface treatment on the CF, so that load can be better transferred from PE to $\mathrm{CF}$, allowing composite materials to withstand greater loads. On the other hand, due to the significant mechanical strength of CNTs, it is generally believed that the incorporation of CNTs mixed into polymer matrix will result in a great improvement in strength. Further evidence for enhancement of CNT-coated CF interfacial adhesion will be presented in SEM studies.

\subsection{Morphology of the composites}

The morphological characterization of $\mathrm{CNT} / \mathrm{CF} / \mathrm{PE}$ composites was investigated through SEM imaging technique in this study. The information on orientation, distribution, surface conditions of CFs and CNTs as well as the interfacial adhesion between the PE matrix and reinforcements were 
correspondingly recorded. Figure 1 (a) and (b) shows the morphologies of CFs and CNTs at the magnification of $5 \mathrm{KX}$ and $100 \mathrm{KX}$ respectively. The surface of CF was extremely smooth, which indicated particularly poor interfacial adhesion between polymer matrix and CFs, and thus resulted in low mechanical performance of CF reinforced PE composites consequently. Therefore, CNTs has been widely utilized in various surface treatments such as spray coating, electrophoretic deposition, chemical grafting, containing sizing etc. to enhance the surface roughness of CFs.

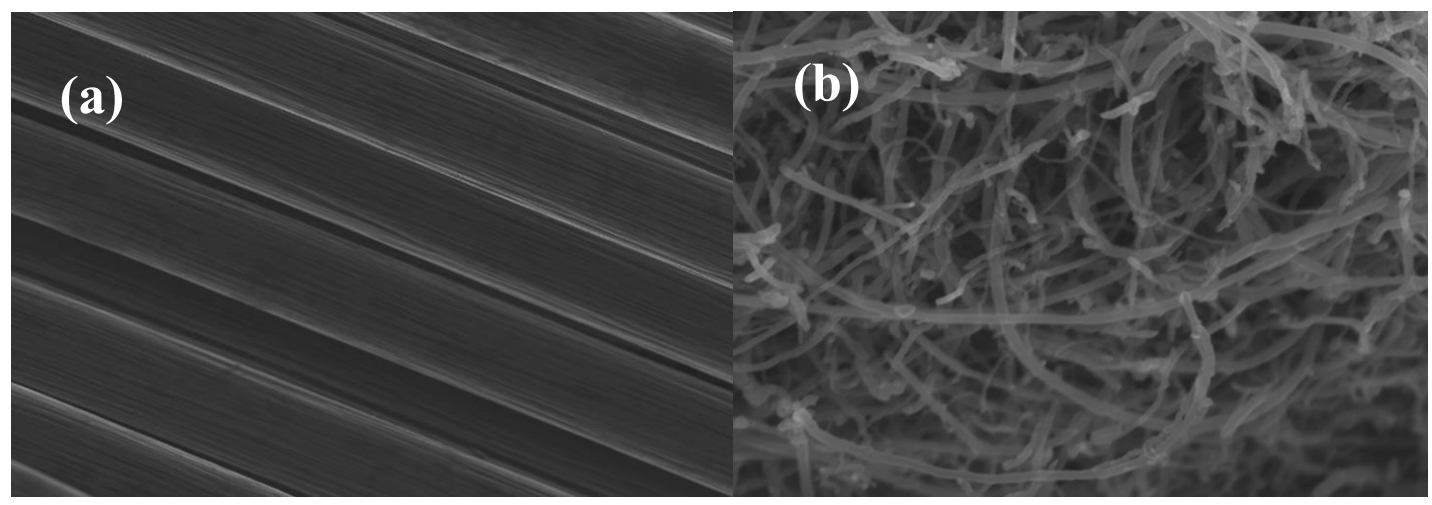

Figure 1. The morphological characterization of (a) CFs and (b) CNTs

In this study, spray coating surface treatment was applied to optimize the surface condition of CFs. Figure 2 (a) exhibited the morphology for CFs after coating with CNTs at the magnification of 100KX. It was obvious to see that the initial smooth homogeneous surface of CF became rougher after the non-uniformly CNTs layer wrapped onto the CF surface and thus CNFs coating on the CF surface was ascertained. The fracture surface of CNTs-coated CF/PE composites were also examined which was shown in Figure 2 (b). As it can be seen, with the roughness increase of CF surface after CNT coating, the PE matrix was attached onto CFs, eliminating the voids normally existing between the matrix and reinforcement. It contributes to the enhancement of interfacial bonding between these two phases, which was verified by the tensile test.

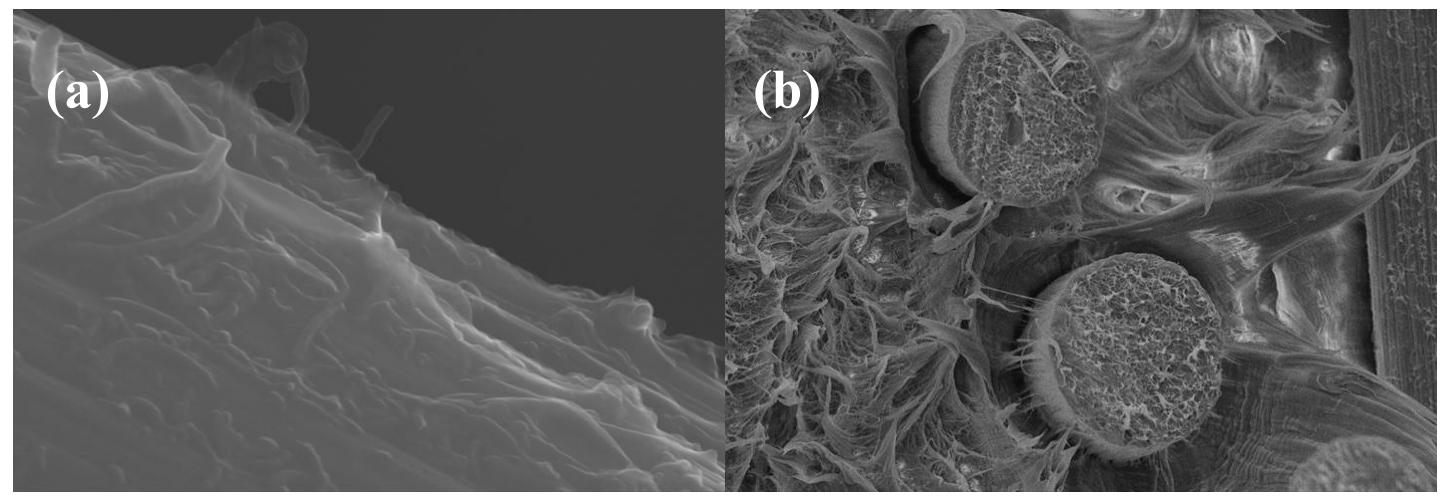

Figure 2. The morphological characterization of (a) CF surface after CNT coating and (b) fractural surface of $\mathrm{CNT} / \mathrm{CF} / \mathrm{PE}$ composites

\section{Conclusions}

In this research, the hierarchical $\mathrm{CNT} / \mathrm{CF} / \mathrm{PE}$ composites were first fabricated by combining the traditional spray coating method with twin-screw extrusion technique. Two groups of composites with or without CNT coatings were compared in terms of mechanical and morphological properties. The results showed that the tensile strength and modulus exhibited an upward tendency for these two 
groups with the rise of $\mathrm{CF}$ content. In particular, the incorporation of CNTs into CF/PE composites contributes to a greater improvement in tensile properties, indicating the enhancement of interfacial adhesion between the reinforcement and matrix. As the most promising technique for future scale-up production, the integration of spray coating with conventional extrusion method will pave the way for the following large-scale industrialization.

\section{References}

1. An, Q., et al., ACS Appl. Mat. Interfaces 5(6): 2022-2032.(2013)

2. Godara, A., et al., Compos. Sci. Technol. , 70(9): 1346-1352.(2010)

3. Chandrasekaran, V., et al., Carbon, 48(13): 3692-3699.(2010)

4. Fan, Z., et al., Composites Part A 39(3): 540-554.(2008)

5. Chang, L. and Z. Zhang, Wear, 260(7): 869-878.(2006)

6. Zhao, S., et al., Compos. Sci. Technol. , 68(14): 2965-2975.(2008)

7. Chen, C. and A. B. Morgan, Polymer, 50(26): 6265-6273.(2009)

8. De Volder, M. F., et al., science, 339(6119): 535-539.(2013)

9. An, F., et al., Mater. Des. , 33: 197-202.(2012)

10. Li, M., et al., Carbon, 52: 109-121.(2013)

11. Chou, T.-W., et al., Compos. Sci. Technol. , 70(1): 1-19.(2010)

12. Kim, M., et al., Compos. Sci. Technol. , 69(3): 335-342.(2009)

13. Díez-Pascual, A. M., et al., Curr. Opin. Solid State Mater. Sci. , 18(2): 62-80.(2014)

14. Alsawat, M., et al., Carbon, 106: 295-305.(2016)

15. Rider, A. N., et al., MRS Advances, 1(12): 785-790.(2016)

16. Withers, G., et al., J. Compos. Mater., 50(6): 783-793.(2016)

17. Zhang, Q., et al., Compos. Sci. Technol. , 69(5): 594-601.(2009)

18. Lee, S.-B., et al., Composites Part A 42(4): 337-344.(2011)

19. Gao, S., et al., Acta Mater. , 55(3): 1043-1052.(2007)

20. Siddiqui, N. A., et al., Composites Part A 41(4): 539-548.(2010)

21. Zhang, H., et al., Composites Part A 70: 102-110.(2015) 